\title{
Plasmablastic lymphomas and plasmablastic plasma cell myelomas have nearly identical immunophenotypic profiles
}

\author{
Francisco Vega ${ }^{1}$, Chung-Che Chang ${ }^{2}$, Leonard J Medeiros ${ }^{3}$, Mark M Udden ${ }^{4}$, \\ Jeong Hee Cho-Vega ${ }^{5}$, Ching-Ching Lau ${ }^{6}$, Chris J Finch ${ }^{1}$, Regis A Vilchez ${ }^{4,7}$, \\ David McGregor ${ }^{1}$ and Jeffrey L Jorgensen ${ }^{3}$
}

\begin{abstract}
${ }^{1}$ Department of Pathology, Baylor College of Medicine, University of Texas MD Anderson Cancer Center, Houston, TX, USA; ${ }^{2}$ Department of Hematopathology, The Methodist Hospital, University of Texas MD Anderson Cancer Center, Houston, TX, USA; ${ }^{3}$ Department of Hematopathology, University of Texas MD Anderson Cancer Center, Houston, TX, USA; ${ }^{4}$ Department of Medicine, Baylor College of Medicine, University of Texas MD Anderson Cancer Center, Houston, TX, USA; ${ }^{5}$ Department of Molecular Pathology, University of Texas MD Anderson Cancer Center, Houston, TX, USA; ${ }^{6}$ Department of Pediatrics, Baylor College of Medicine, University of Texas MD Anderson Cancer Center, Houston, TX, USA and ${ }^{7}$ Department of Molecular Virology and Microbiology, Baylor College of Medicine, University of Texas MD Anderson Cancer Center, Houston, TX, USA
\end{abstract}

\begin{abstract}
Plasmablastic lymphoma is an aggressive neoplasm that shares many cytomorphologic and immunophenotypic features with plasmablastic plasma cell myeloma. However, plasmablastic lymphoma is listed in the World Health Organization (WHO) classification as a variant of diffuse large B-cell lymphoma. To characterize the relationship between plasmablastic lymphoma and plasmablastic plasma cell myeloma, we performed immunohistochemistry using a large panel of B-cell and plasma cell markers on nine cases of plasmablastic lymphoma and seven cases of plasmablastic plasma cell myeloma with and without HIV/AIDS. The expression profiles of the tumor suppressor genes p53, p16, and p27, and the presence of Epstein-Barr virus (EBV) and human herpes virus type 8 (HHV-8) were also analyzed. All cases of plasmablastic lymphoma and plasmablastic plasma cell myeloma were positive for MUM1/IRF4, CD138, and CD38, and negative for CD20, corresponding to a plasma cell immunophenotype. PAX-5 and BCL-6 were weakly positive in $2 / 9$ and $1 / 5$ plasmablastic lymphomas, and negative in all plasmablastic plasma cell myelomas. Three markers that are often aberrantly expressed in cases of plasma cell myelomas, CD56, CD4 and CD10, were positive in 5/9, 2/5, and 6/9 plasmablastic lymphomas, and in 3/7, 1/5, and 2/7 plasmablastic plasma cell myelomas. A high Ki-67 proliferation index, overexpression of p53, and loss of expression of p16 and p27 were present in both tumors. No evidence of HHV-8 infection was detected in either neoplasm. The only significant difference between plasmablastic lymphoma and plasma cell myeloma was the presence of EBV-encoded RNA, which was positive in all plasmablastic lymphoma cases tested and negative in all plasma cell myelomas. In conclusion, most cases of AIDS-related plasmablastic lymphoma have an immunophenotype and tumor suppressor gene expression profile virtually identical to plasmablastic plasma cell myeloma, and unlike diffuse large B-cell lymphoma. These results do not support the suggestion in the WHO classification that plasmablastic lymphoma is a variant of diffuse large B-cell lymphoma.

Modern Pathology (2005) 18, 806-815, advance online publication, 3 December 2004; doi:10.1038/modpathol.3800355
\end{abstract}

Keywords: AIDS-related lymphomas; EBV infection; plasmablastic lymphoma; plasmablastic plasma cell myeloma

Correspondence: Dr C-C Chang, MD, PhD, Department of Hematopathology, The Methodist Hospital, 6565 Fannin, MS205, Houston, TX 77030, USA.

E-mail: jeffchang@tmh.tmc.edu

This work was presented, in part, at the 2004 Annual Meeting of the United States and Canadian Society of Pathology, in Vancouver, Canada.

Received 15 September 2004; revised and accepted 27 October 2004; published online 3 December 2004
In 1997, Delecluse et $a l^{1}$ described an AIDSassociated B-lineage neoplasm with plasmacytic differentiation, typically presenting in the oral cavity, and proposed that this constituted a new subtype of diffuse large B-cell lymphoma, for which they coined the term plasmablastic lymphoma. Plasmablastic lymphoma is considered a variant of diffuse large B-cell lymphoma in the World Health 
Organization (WHO) classification of lymphoid neoplasms. ${ }^{2}$

The diagnosis of plasmablastic lymphoma can be complicated by its morphologic resemblance to other lymphoid and myeloid malignancies. Immunoblastic diffuse large B-cell lymphoma can be excluded on the basis of the characteristic immunophenotypic pattern of plasmablastic lymphoma, with CD20 negativity in combination with markers of postgerminal center B cells and plasma cells, such as CD138/syndecan-1. ${ }^{3,4}$ Diffuse large B-cell lymphoma with expression of ALK and extramedullary myeloid tumor can be excluded by immunohistochemical studies for ALK and myeloid markers, respectively. $^{5,6}$ However, in many cases of plasmablastic lymphoma, the most difficult issue in the differential diagnosis is excluding plasmablastic plasma cell myeloma with extramedullary involvement. This is a clinically important distinction, since plasmablastic lymphoma is listed in the WHO classification as a variant of diffuse large B-cell lymphoma implying that therapy for diffuse large B-cell lymphoma is appropriate, while plasma cell myeloma cases are treated with different chemotherapy regimens.

Morphological features can be used to distinguish plasmablastic lymphoma from well-differentiated plasma cell neoplasms. However, highly aggressive plasma cell myeloma may contain a predominance of plasmablasts, which can closely resemble the malignant cells of plasmablastic lymphoma. ${ }^{7}$ Previous reports have shown that plasmablastic lymphoma has a terminally differentiated B-cell or plasma cell immunophenotype, characterized by weak or absent expression of conventional B-cell markers, and strong expression of the post germinal center B-cell- and plasma cell-associated markers MUM1/IRF4 and CD138/syndecan-1. ${ }^{1-3,8,9}$ This immunophenotype is similar to that reported for plasmablastic plasma cell myeloma. ${ }^{1,8,10,11}$

In practice, the distinction between plasmablastic lymphoma and plasmablastic plasma cell myeloma frequently depends on clinical correlation. The presence of serum monoclonal proteins, and/or bone involvement with radiographically evident lytic lesions, favors the diagnosis of plasma cell myeloma rather than plasmablastic lymphoma. In addition, the presence of Epstein-Barr virus (EBV) infection is much more strongly associated with plasmablastic lymphoma than with plasma cell neoplasms. ${ }^{12}$ Plasma cell myeloma (and plasmacytoma) may occasionally occur in the setting of AIDS; ${ }^{13,14}$ however, they do not have the strong association with AIDS that has been reported for plasmablastic lymphoma.

Our goal in this study was to further define the relationship between plasmablastic lymphoma and plasmablastic plasma cell myeloma by assessing the immunophenotypic features of these neoplasms, using a large panel of lymphoid and plasma cellrelated markers. Our panel included CD4, CD10, and CD56, in order to assess whether these antigens may be aberrantly expressed in both types of neoplasm. We also analyzed for expression of the tumor suppressor gene products p16, p27, and p53, as well as for the presence of EBV and human herpes virus type 8 (HHV-8).

\section{Materials and methods}

\section{Histologic Features}

The diagnosis of plasmablastic lymphoma was based on the criteria described by the WHO classification of lymphoid neoplasms. ${ }^{2}$ We subdivided the plasmablastic lymphoma cases into immunoblastic or plasmablastic types, based on the predominant cellular component. Plasmablasts were distinguished from immunoblasts by their smaller size, more rounded nuclei, coarser chromatin, and smaller nucleoli. ${ }^{15}$ The diagnosis of plasma cell myeloma was also based on the morphologic, clinical, and laboratory criteria described by the WHO classification of lymphoid neoplasms. ${ }^{16} \mathrm{We}$ selected only morphologically high-grade plasma cell myeloma cases, with a predominant plasmablastic component.

\section{Immunohistochemistry}

Immunohistochemical stains were performed using formalin-fixed paraffin-embedded tissue sections and an automated immunostainer (DAKO, Carpenteria, CA, USA). ${ }^{17}$ All tissue sections underwent heat-induced antigen retrieval. The antibodies used were specific for Ki-67 (1:50, MIB-1), CD20 (1:1000, L26), CD79a (1:50, JCB117), CD30 (1:200, Ki-1), ALK-1 (1:50), P53 (1:500, DO-7), BCL-6 (1:200, PGB6P), $\kappa$ (1:250 000), $\lambda$ (1:250 000), EBV-LMP-1 (1:100, CS1-4), and CD45 (1:1000) (DAKO); CD4 (1:300, 4B12), CD38 (1:100, SPC32), BCL-2 (1:200), and HHV-8 (1:100) (Novocastra, Newcastle, UK); PAX-5 (1:200) (Transduction Labs, San Diego, CA, USA); CD138 (1:200, B-B4) (Serotec, Raleigh, NC, USA); CD56 (1:150, 123C3) (Zymed, San Francisco, CA, USA); IRF-4 (1:400, MUM-1) and CD117 (1:400) (Santa Cruz, Santa Cruz, CA, USA); p27 (1:200, Kip1) (Cell Signaling, Beverly, MA, USA); and CD3 (1:40, 1F6), CD10 (1:50, 56C6), and p16 (1:50, JC8), (Lab Vision, Fremont, CA, USA). Some of these immunohistochemical stains had been performed as part of diagnostic work up in individual cases to establish the diagnosis and were reviewed for this study. Envision Plus Labeled Polymers for mouse or rabbit (DAKO) were utilized for detection, with diaminobenzidine as the chromogenic substrate. A case was scored as positive for each marker (including the tumor suppressor markers) if immunostaining was obtained in more than $10 \%$ of the neoplastic cells with appropriate localization and with appropriate staining of controls. For example, the absence of nuclear staining was considered to be a loss of p16 and p27 when the interspersed nuclei 
of reactive lymphocytes, endothelial cells, or fibroblasts displayed p16 or p27 expression. Some markers were not assessed in all cases because of a limited amount of tissue.

\section{In Situ Hybridization}

In situ hybridization analysis for EBV was performed on formalin-fixed paraffin-embedded tissue sections using a Novocastra ISH kit (NCL-EBV-K) according to the manufacturer's instructions with the appropriate positive and negative controls.

\section{Polymerase Chain Reaction Amplification Method}

Samples were analyzed by polymerase chain reaction (PCR) for the presence of HHV-8 DNA. Oligonucleotide primers have been described previously. ${ }^{18,19}$ All of the DNA samples were tested for suitability for amplification using primers specific for a fragment of the human $\beta$-hemoglobin gene (primers PC03/KM38). Only specimens from which cellular $\beta$-globin gene sequences were amplified were then examined by PCR amplification using primers KS-1, 5'-AGC CGA AAG GAT TCC ACC AT$3^{\prime}$ and KS-2, $5^{\prime}$-TTC GTG TTG TCT ACG TCC AG-3' targeting a 233-bp fragment of the open reading frame $26 .{ }^{18}$ Primers were obtained from Integrated DNA Technologies, Inc. (Coralville, IA, USA). The positive control was a plasmid DNA containing cloned viral sequences of the HHV-8 capsid antigen gene. Negative controls for PCR assays were reactions without added DNA template. PCR amplifications were performed with a Perkin-Elmer GeneAmp PCR system 2400 thermocycler. High-stringency annealing temperatures specific for each primer set have been described elsewhere. ${ }^{18,19}$ PCR amplification products were analyzed by agarose gel electrophoresis following standard protocols.

\section{Statistical Analysis}

Differences in the immunophenotype profiles between plasmablastic lymphoma and plasmablastic plasma cell myeloma were analyzed by Fisher's exact test.

\section{Results}

\section{Clinical Features}

Nine cases of plasmablastic lymphoma, in eight males and one female, and seven cases of plasmablastic plasma cell myeloma, in three males and four females, were identified from the files of the pathology departments at The Methodist Hospital, Ben Taub General Hospital, and MD Anderson Cancer Center, Houston, Texas, from 1999 to 2004. All patients with plasmablastic lymphoma were HIV positive. The age at diagnosis ranged between 23 and 58 years. Seven cases presented in the oral cavity or nasopharinx and two cases involved an extra-oral site (anus and supraclavicular lymph nodes) (Table 1). The tumors in the oral cavity involved predominantly the oral mucosa although radiographically involvement of the subjacent bone was seen in three cases (Table 1). Review of the available clinical information showed that none of the patients with plasmablastic lymphoma had monoclonal gammopathy, multiple osteolytic bone lesions, or a prior history of a plasma cell myeloma (or plasmacytoma).

The clinical features of the patients with plasmablastic plasma cell myeloma are detailed in Table 1. The age at diagnosis ranged between 37 and 76 years. The diagnosis of plasma cell myeloma was supported by clinical and/or laboratory data. Six patients had multiple osteolytic lesions (cases 2-7) and four patients had a monoclonal paraprotein in serum and urine (cases 1,5-7). Two patients had a previous history of plasma cell myeloma (cases 5 and 6). Two patients were HIV positive (cases 1 and 4) and five were HIV negative (cases 2, 3, 5-7).

\section{Histologic Findings}

All plasmablastic lymphoma cases showed similar histological features, consisting of a diffuse proliferation of predominantly plasmablasts (five cases) or immunoblasts (four cases), with relatively few morphologic features of mature plasmacytic differentiation (Figure 1a and b). Apoptotic bodies and mitotic figures were numerous, and confluent areas of necrosis were occasionally identified. A starrysky background with tingible-body macrophages was seen in six plasmablastic lymphoma cases. Small mature $\mathrm{T}$ cells $(\mathrm{CD} 3+)$ were interspersed among the tumor cells. In two plasmablastic lymphoma cases, numerous neoplastic multinucleated cells were present. Smaller neoplastic cells with obvious plasmacytic differentiation were rare, representing less than $1 \%$ of cells in all the cases.

All seven plasmablastic plasma cell myeloma cases showed a diffuse infiltrate of medium-sized tumor cells, most showing plasmablastic features, with a subset of cells showing immunoblastic features (Figure 2a and b). One case (case 7) showed a higher proportion of immunoblasts, but plasmablasts were still the predominant component. Variable numbers of smaller cells with obvious plasmacytic differentiation were seen in all cases, intermingled with the larger plasmablasts. Apoptotic bodies and mitotic figures were frequent. Small areas of necrosis were seen in one case. A focal starry-sky background with tingible body macrophages was seen in two cases. Russell bodies were present in two cases. None of the plasmablastic plasma cell myeloma contained amyloid deposits. No significant morphological differences were 
observed between plasmablastic plasma cell myeloma in HIV-positive vs HIV-negative patients.

\section{Immunophenotypic Analysis}

The results of immunohistochemical studies are summarized in Table 2. In all plasmablastic lymphoma cases, the neoplastic cells showed strong expression of the post-germinal center B-cell/plasma cell-associated antigens MUM-1/IRF4, CD38, and CD138/syndecan-1. CD10 was positive in 6/9, CD56 in 5/9, and CD4 in 2/5 plasmablastic lymphoma cases; eight of nine cases were positive for one or more of those antigens. Three of eight cases were positive for BCL-2. Monotypic light chain expression was demonstrated by immunohistochemistry in three of seven cases. PAX-5 was weakly positive in two of nine, CD79a was positive in one of six and BCL-6 was weakly positive in one of five cases. All cases assessed were negative for CD3, CD20, CD30, CD117, and ALK-1. The proliferative index, as determined by Ki-67 staining, was greater than $70 \%$ in all cases, with a median of $87 \%$. All eight cases assessed were positive for $\mathrm{p} 53$ and negative for p16. Loss of p27 expression was seen in two of four cases. We found no significant differences in frequency of expression of any immunohistochemical marker between plasmablastic lymphoma composed predominantly of immunoblasts vs plasmablastic lymphoma composed predominantly of plasmablasts.

The plasmablastic plasma cell myeloma cases had an immunophenotypic profile virtually identical to that of the plasmablastic lymphoma cases. In all seven plasmablastic plasma cell myeloma cases, the neoplastic cells showed strong expression of MUM1/IRF4, CD38, and CD138/syndecan-1. CD10 was positive in 2/7, CD56 in 3/7, and CD4 in 1/5 cases; in total, five of seven plasma cell myeloma cases were positive for one or more of these three markers (Figure 2). Monotypic light chain expression was demonstrated by immunohistochemistry in six of seven cases. Four of seven cases were positive for BCL-2, and one of seven cases was positive for CD117. All cases assessed were negative for CD3, CD20, CD30, CD79a, ALK-1, BCL-6, and PAX-5. Five of seven cases were positive for p53. All cases assessed were negative for $\mathrm{p} 16$, and four of five cases were negative for $\mathrm{p} 27$. The proliferative index was variable (range $40-95 \%$ ) with a median of $79 \%$. We found no statistically significant difference in frequency of expression of any immunohistochemical marker between plasmablastic lymphoma and plasmablastic plasma cell myeloma cases.

\section{Analysis of Viral Infection}

Infection by EBV, as defined by in situ hybridization for EBV-encoded RNA (EBER), was found in all five plasmablastic lymphoma cases analyzed (Table 2 

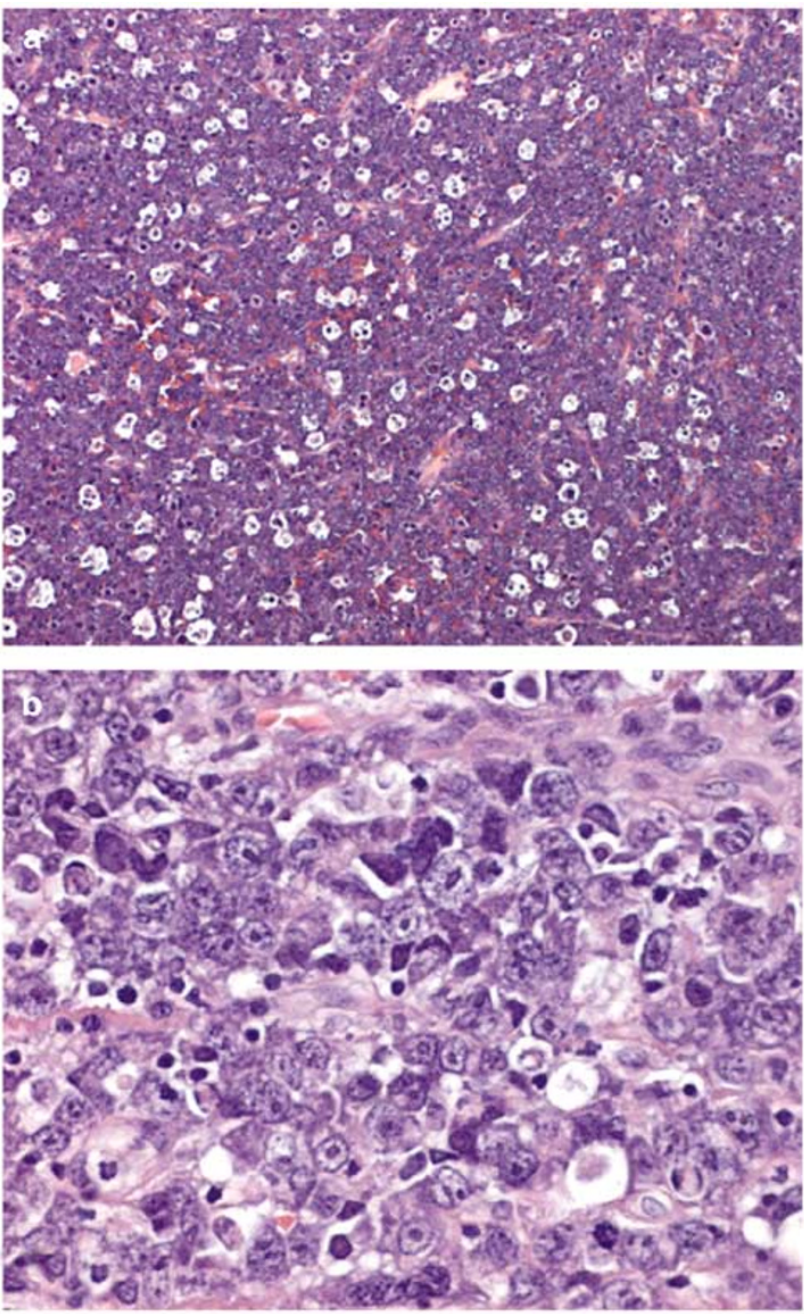

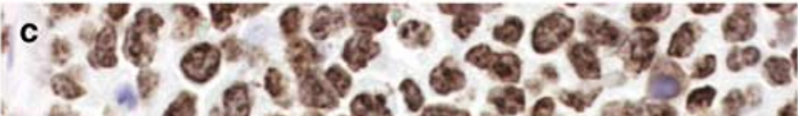

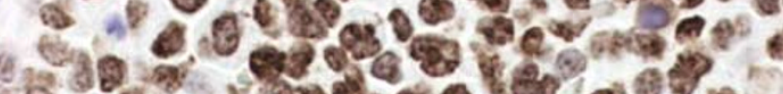

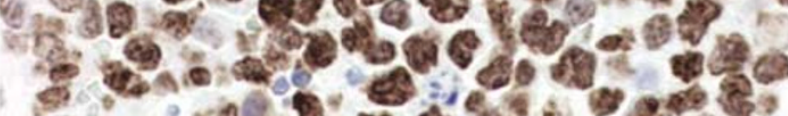

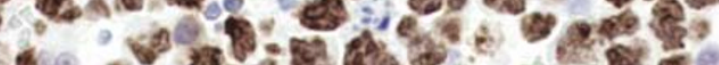

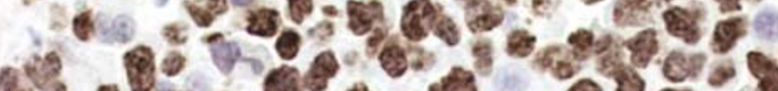

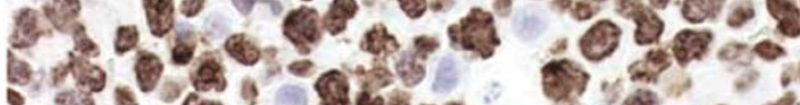
2. 4he 6.

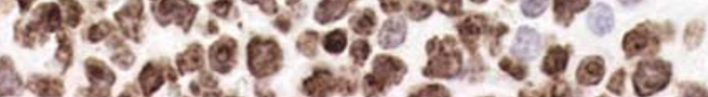
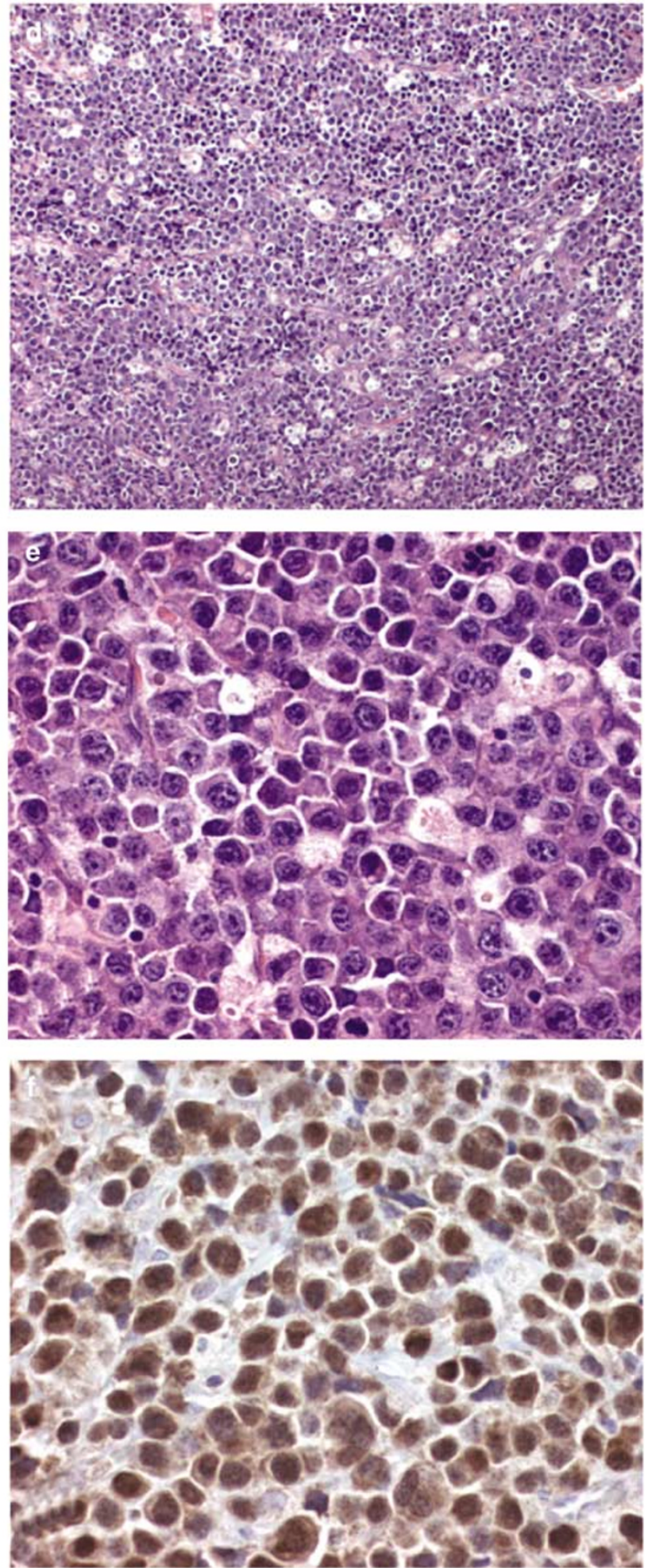

Figure 1 Morphologic features and proliferation index of plasmablastic lymphoma and plasmablastic plasma cell myeloma. (a) Plasmablastic lymphoma, case 6. Diffuse infiltrate of large cells, with a starry-sky pattern of tingible body macrophages (H\&E, $\times 40$ ). (b) Plasmablastic lymphoma, case 7 . The tumor cells have features of immunoblasts; they are large with a vesicular chromatin and frequently prominent central nucleoli. Rare smaller neoplastic cells with plasmacytic differentiation are also noted (H\&E, $\times 400)$. (c) Plasmablastic lymphoma, case 6. The proliferation index, as determined by Ki-67, was higher than $90 \%(\mathrm{Ki}-67$, $\times 400)$. (d) Plasmablastic plasma cell myeloma, case 1. Diffuse infiltrate characterized by a focal starry-sky background (H\&E, original magnification $\times 40$ ). (e) Plasmablastic plasma cell myeloma, case 1 . The tumor cells have marked plasmacytic differentiation. Larger atypical cells with features of plasmablasts are interspersed among the plasmacytic cells (H\&E, original magnification $\times 400$ ). (f) Plasmablastic plasma cell myeloma, case 1 . The proliferation index was higher than $90 \%(\mathrm{Ki}-67, \times 400)$. 
and Figure 2). In contrast, EBER was not detected in any of the seven plasmablastic plasma cell myelomas (5/5 vs 0/7, $P<0.002$, Fisher's-exact test) (Figure 2). EBV LMP-1, assessed by immunohistochemistry in three plasmablastic lymphomas and all seven plasma cell myelomas, was negative in all cases. HHV-8 was negative by immunohistochemistry and PCR in all the cases of plasmablastic lymphoma and plasmablastic plasma cell myeloma assessed (Table 2).

\section{Discussion}

Previous studies have described an overlap in cytomorphologic and immunophenotypic features between plasmablastic lymphoma and plasmablastic plasma cell myeloma. ${ }^{1,8,10,11,15}$ In practice, it may be very difficult to distinguish these neoplasms using solely morphologic criteria. Thus, in this study we used clinical and laboratory data as well as pathological findings to identify plasma cell myeloma cases, and then performed a comprehensive panel of lymphoid and plasma cell-related markers, to compare plasmablastic lymphoma and plasmablastic plasma cell myeloma.

In this study, plasmablastic lymphoma and plasmablastic plasma cell myeloma cases were morphologically quite similar. However, there were some subtle differences in morphology. We found that the number of cells with obvious features of plasma cell maturation, including eccentric nuclei, abundant basophilic cytoplasm, and paranuclear hofs, differed between these neoplasms. Plasmablastic lymphomas were characterized by a monotonous proliferation of plasmablasts and/or immunoblasts, with rare or no obvious plasmacytic cells, whereas plasmacytic cells were frequently seen in plasmablastic plasma cell myeloma. Similar morphological differences were also reported by Colomo et al ${ }^{15}$ In practice, these differences may not be sufficient to reliably establish a diagnosis in an individual case.

Our results show a virtually identical immunophenotypic profile for plasmablastic lymphoma and plasmablastic plasma cell myeloma. In agreement with previous studies, all of the plasmablastic lymphoma cases in this study had a post-germinal center B-cell/plasma cell phenotype, expressing MUM1/IRF4 and CD138/syndecan-1, but negative for CD20., ${ }^{3,20-22}$ Furthermore, we observed that several markers that may be aberrantly expressed in plasma cell neoplasms, CD56, CD10 and CD4, were also expressed in most cases of plasmablastic lymphoma. CD56 and CD10, markers frequently expressed in plasma cell myeloma, ${ }^{23-25}$ were positive in 5/9 and in 6/9 of the plasmablastic lymphoma cases in our series, respectively. Given the preponderance of post-germinal center markers seen in all of our plasmablastic lymphoma cases, we believe that CD10 expression in plasmablastic lymphoma is temporally aberrant, as may be seen in plasma cell myeloma. Two cases of plasmablastic lymphoma were positive for CD4, which is aberrantly expressed in some plasma cell myelomas. ${ }^{24,25}$ Overall, all plasmablastic lymphoma cases except one expressed one or more of these three markers. As expected, each of these markers was also positive in a subset of cases of plasmablastic plasma cell myeloma.

By contrast, the immunophenotype of the plasmablastic lymphoma cases was unlike that of diffuse large B-cell lymphoma as reported in the literature. Diffuse large B-cell lymphomas usually express the pan-B-cell antigens, CD20, CD79a and PAX-5, ${ }^{22,26}$ and frequently express BCL-6 (ranging from 50 to $80 \%$ of the cases). ${ }^{27-29}$ Our plasmablastic lymphoma cases were consistently negative for CD20, and almost all cases were negative for CD79a, BCL-6, and PAX-5. PAX-5, a B-cell-specific transcription factor, is detectable as early as the pro-B-cell stage and subsequently in all further stages of B-cell development until the plasma cell stage, where it is downregulated. ${ }^{30,31}$ The expression of PAX-5 is generally absent in plasma cell malignancies and is present in nearly all cases of diffuse large B-cell lymphoma. ${ }^{22}$ To our knowledge, PAX-5 expression has not been studied previously in plasmablastic lymphoma. The absence of PAX-5 expression demonstrated in our study in nearly all plasmablastic lymphoma cases provides additional evidence for plasma cell differentiation in plasmablastic lymphoma. Moreover, most of our plasmablastic lymphoma cases were positive for CD56 and almost the half of the cases studied were positive for CD4. CD56 expression in diffuse large B-cell lymphoma is rare, and some have argued that CD56 positivity should exclude the diagnosis of lymphoma. ${ }^{32-34}$ CD4 expression is extremely rare in diffuse large B-cell lymphomas. ${ }^{35}$

One plasmablastic lymphoma in our series showed focal weak expression of PAX-5 (case 3) and another plasmablastic lymphoma showed weak positivity for both PAX-5 and BCL-6, but was negative for CD10 (case 9). These findings suggest that these two cases may reflect a slightly earlier, perhaps immunoblastic stage of B-lineage differentiation, compared with the other plasmablastic lymphoma cases in our series. However, these two cases otherwise demonstrated a terminally differentiated B-cell/plasma cell phenotype (CD20-, CD38 +, CD138+, MUM-1 +), which as noted above would be a very unusual pattern for diffuse large B-cell lymphoma.

The frequency of CD56 expression in plasmablastic lymphoma cases in this study appears to differ from that of Colomo et al, ${ }^{15}$ who studied a series of large B-cell lymphomas with plasmablastic differentiation. Their approach to classifying these cases differed from the approach we used in this study. They divided their cases on the basis of morphologic features (primarily the presence or absence of more differentiated plasmacytic cells), and then 
CD56

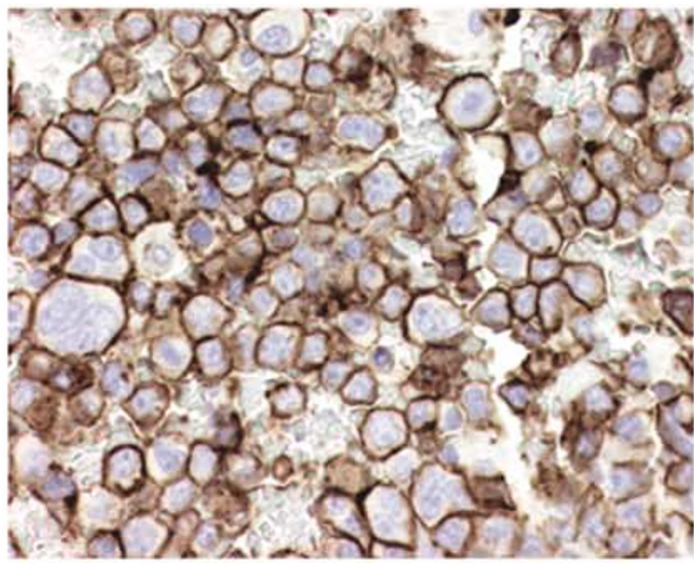

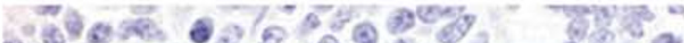

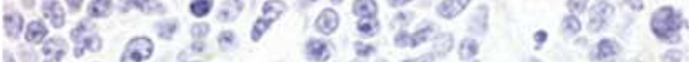

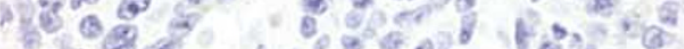

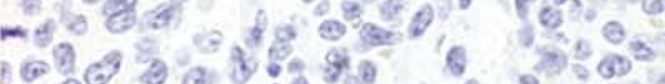

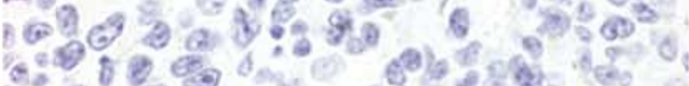

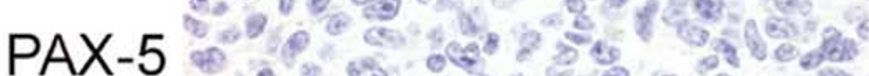

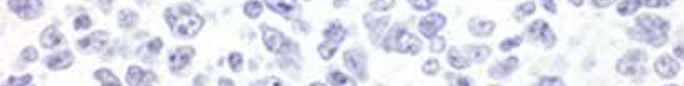

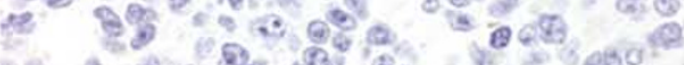
F. \& \& अ

CD4
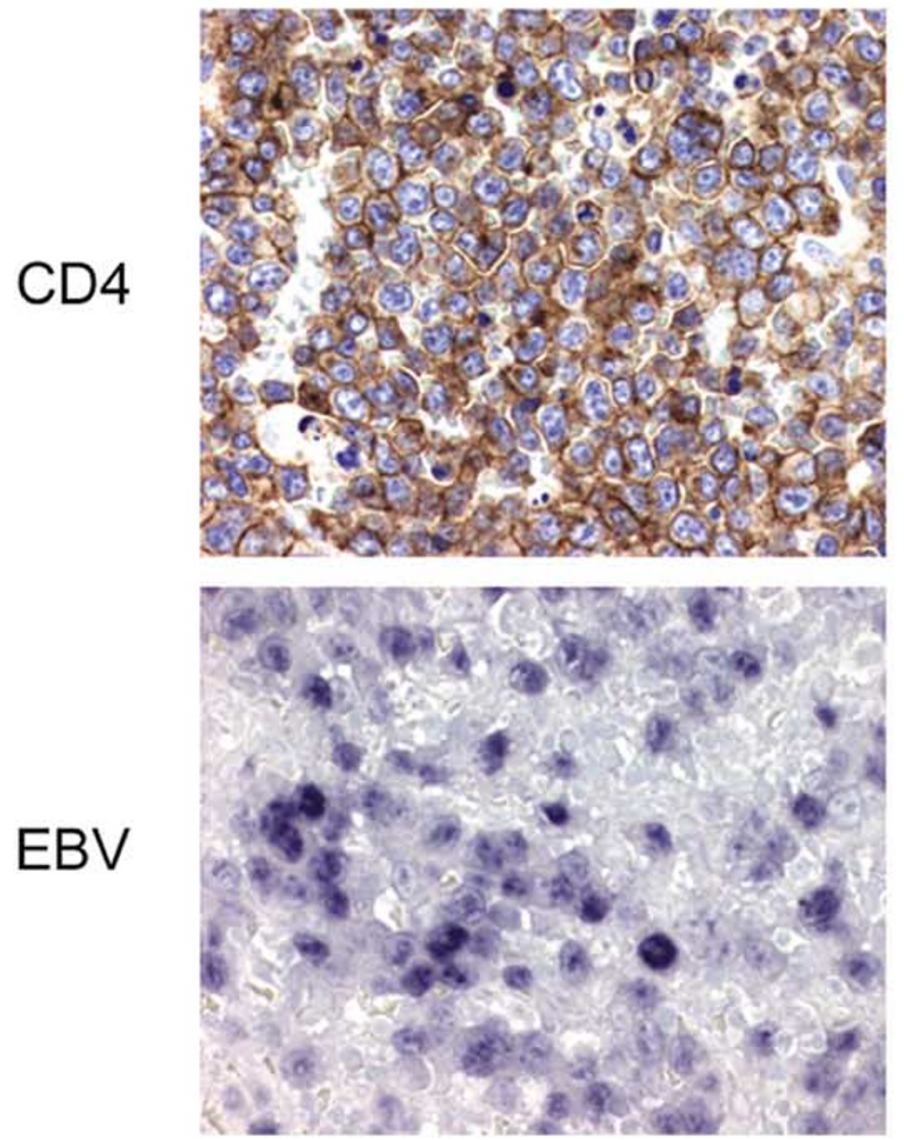

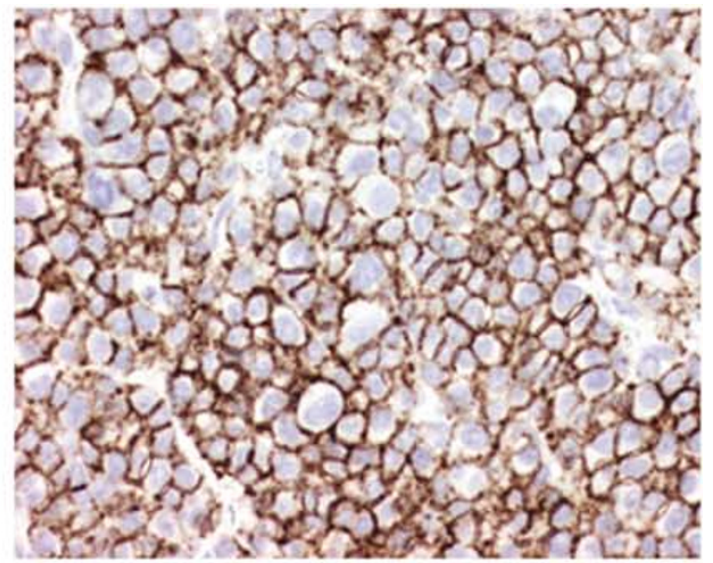

का क्ष०त

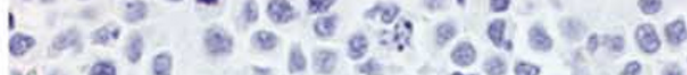

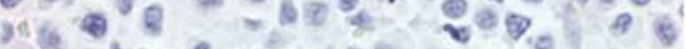

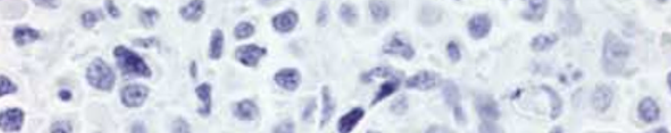

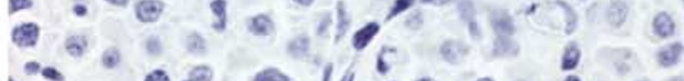

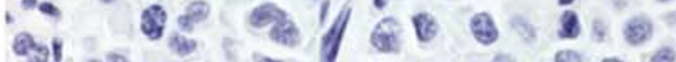

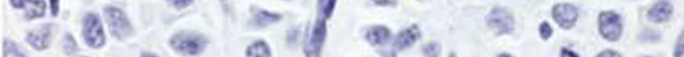

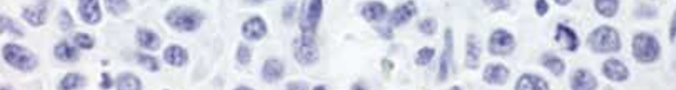

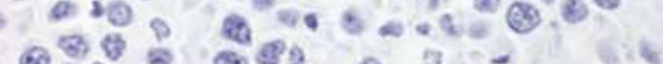

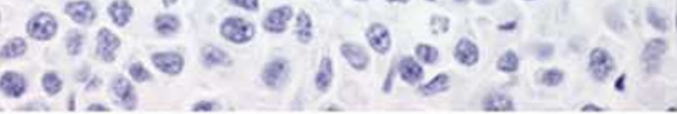
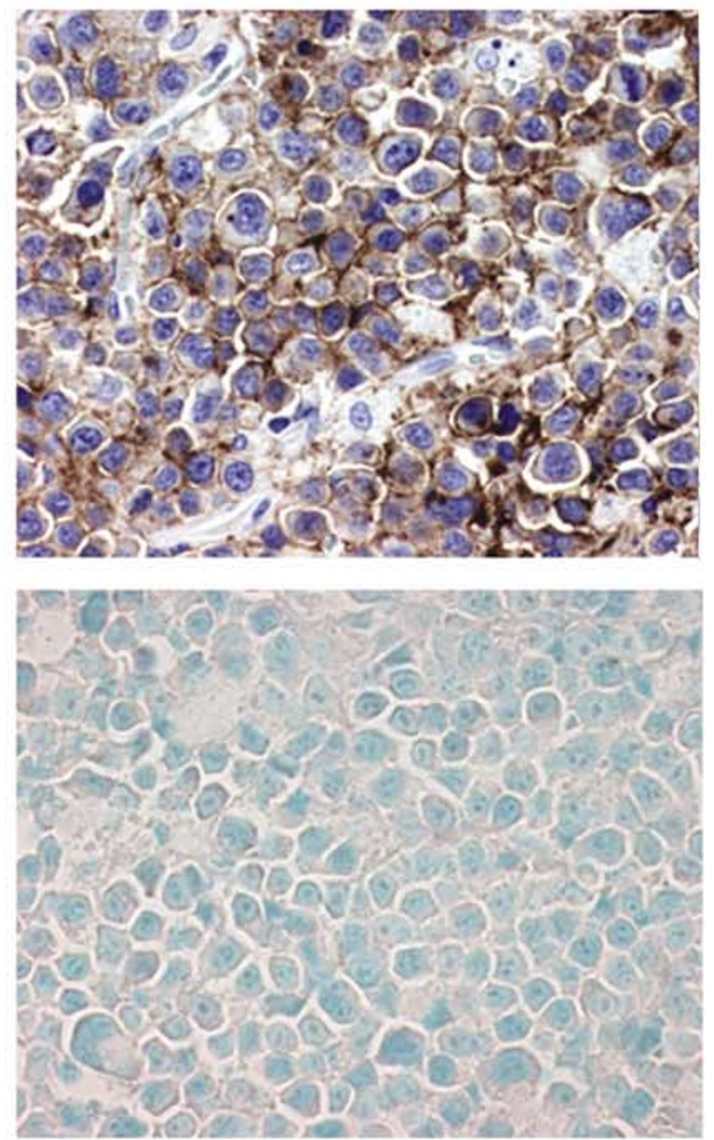
Table 2 Comparison of immunophenotypic, tumor suppressor gene and viral profiles between plasmablastic lymphoma and plasmablastic plasma cell myeloma

No. of positive cases/no. of total cases assessed (\%)

Plasmablastic Plasmablastic plasma cell

lymphoma $\mathrm{n}=9 \quad$ myeloma $\mathrm{n}=7$

\begin{tabular}{lcc}
\hline LCA & $6 / 9(67$ & $2 / 6(33)$ \\
CD20 & $0 / 9$ & $0 / 7$ \\
PAX-5 & $2 / 9(22)^{\mathrm{a}}$ & $0 / 6$ \\
CD79a & $1 / 6(17)$ & $0 / 2$ \\
BCL-6 & $1 / 5(20)^{\mathrm{a}}$ & $0 / 7$ \\
CD138 & $9 / 9(100)$ & $7 / 7(100)$ \\
CD38 & $9 / 9(100)$ & $7 / 7(100)$ \\
MUM-1 & $8 / 8(100)$ & $7 / 7(100)$ \\
CD56 & $5 / 9(56)$ & $3 / 7(43)$ \\
CD10 & $6 / 9(67)$ & $2 / 7(29)$ \\
CD4 & $2 / 5(40)$ & $1 / 5(20)$ \\
CD117 & $0 / 4$ & $1 / 7(14)$ \\
CD30 & $0 / 7$ & $0 / 5$ \\
ALK-1 & $0 / 8$ & $0 / 7$ \\
BCL-2 & $3 / 8(38)$ & $4 / 7(57)$ \\
$\kappa / \lambda$ restr & $3 / 7(43)$ & $6 / 7(86)$ \\
Ki-67 & $87^{\mathrm{b}}$ & $79^{\mathrm{b}}$ \\
& & \\
p53 expression & $8 / 8(100)$ & $5 / 7(71)$ \\
Loss of p16 & $8 / 8(100)$ & $5 / 5(100)$ \\
expression & $2 / 4(50)$ & $4 / 5(80)$ \\
Loss of p27 & & \\
expression & & $0 / 7$ \\
EBER & $5 / 5(100)$ & $0 / 7$ \\
LMP-1 & $0 / 3$ & $0 / 6$ \\
HHV-8 & $0 / 6$ & \\
\hline
\end{tabular}

${ }^{\mathrm{a}}$ Weakly and focally expressed. ${ }^{\mathrm{b}}$ Mean value of all the cases.

${ }^{\mathrm{c}}$ Difference statistically significant $(P<0.002$, Fisher's exact test).

${ }^{\mathrm{d}}$ By immunohistochemistry and/or PCR.

determined which clinicopathologic entities fell into those categories. By contrast, we classified our cases as plasmablastic lymphoma or plasma cell myeloma, based on the presence or absence of clinical features of systemic myeloma, then explored the morphologic findings and immunophenotypes of our two sets of cases. Colomo et al ${ }^{15}$ found CD56 expression in only one of 18 cases in their category of plasmablastic lymphoma of oral mucosa type, while we observed CD56 positivity in the majority of our cases of plasmablastic lymphoma (most of which were from the oral cavity or nasopharynx). The basis for this discordance is unclear. The same monoclonal antibody was used in both studies, although from different suppliers; this and other possible technical differences in immunohistochemistry may account for the discrepancy. We also note that their category of plasmablastic lymphoma with plasmacytic differentiation showed CD56 positivity in nearly half of the cases; this category was clinically heterogeneous, and it included several cases from HIV-positive patients, some from the oral cavity or other mucosal sites, with clinical findings similar to the patients in our series. Colomo et al ${ }^{15}$ suggested that CD56 positivity could be used to identify cases of plasmablastic lymphoma secondary to more differentiated plasma cell neoplasms. Our data do not support this proposal, as we observed a number of CD56-positive plasmablastic lymphoma cases with no evidence of systemic myeloma.

In this study, both plasmablastic lymphoma and plasmablastic plasma cell myeloma had a high proliferation index. Moreover, both diseases showed similar tumor suppressor gene expression profiles, with frequent loss of expression of p16 and p27, and positive staining for $\mathrm{p} 53$, the latter suggesting the presence of p53 gene mutation because wild-type p53 is usually undetectable by immunohistochemistry. These findings are not entirely unexpected, because alterations in these tumor suppressors genes may contribute to the highly aggressive clinical behavior of these two neoplasms. Unlike well-differentiated plasma cell neoplasms, plasmablastic plasma cell myelomas usually have an extremely high proliferation index and alterations in cell cycle regulators such as p16, p21, and p53. ${ }^{10,36-38}$

In situ hybridization assays for EBER were positive in all five cases of plasmablastic lymphoma analyzed, and were negative in all plasmablastic plasma cell myelomas. Although the number of cases analyzed is relatively small, the difference in EBER expression between plasmablastic lymphoma and plasma cell myeloma was highly statistically significant $(P<0.002)$. In previously published multicase series of plasmablastic lymphoma, the rate of positivity for EBER ranged from 60 to $86 \% .^{1,20}$ In contrast, positivity for EBER has been reported to be rare in plasma cell myeloma. ${ }^{12}$ Thus, from a practical point of view, when considering a single case, a positive EBER result makes the diagnosis of plasma cell myeloma unlikely. On the other hand, a negative EBER does not completely exclude plasmablastic lymphoma.

In agreement with several previous studies, ${ }^{1,9,15,39,40}$ we did not detect HHV-8 infection in any of the plasmablastic lymphoma or plasmablastic plasma cell myeloma cases assessed using both immunohistochemistry and PCR methodology. Recently, however, Cioc et $a l^{41}$ using RT in situ PCR methods identified HHV-8 infection in four cases of plasmablastic lymphoma involving the oral cavity.

Figure 2 Immunophenotypical profile and EBV status of plasmablastic lymphoma and plasmablastic plasma cell myeloma. Left column, plasmablastic lymphoma, case $6($ CD56, PAX-5 and CD4 $\times 400)$ and case 7 (EBER transcripts, $\times 400)$; right column, plasmablastic plasma cell myeloma, case 6 (CD56, $\times 400)$ and case 1 (PAX-5, CD4 and EBER transcripts, $\times 400$ 
This issue is controversial and further study is needed to address this discordance.

In conclusion, plasmablastic lymphoma has an immunophenotypic profile virtually identical to that of plasmablastic plasma cell myeloma. Even using a comprehensive panel of immunomarkers, as was performed in this study, we cannot reliably distinguish plasmablastic lymphoma from plasmablastic plasma cell myeloma, suggesting a close relationship between these tumors. The main difference we identified was the association of EBV infection with plasmablastic lymphoma, but not with plasmablastic plasma cell myeloma. For these reasons, it may not be appropriate to consider plasmablastic lymphoma as a variant of diffuse large B-cell lymphoma as has been suggested in the WHO classification. However, our concern is based on the pathologic and immunophenotypic data we have presented, and we cannot comment on the optimal therapeutic approach for patients with plasmablastic lymphoma. We would suggest that studies to rule out systemic plasma cell myeloma, including a skeletal survey and serum protein electrophoresis, may be warranted in cases of possible plasmablastic lymphoma, particularly if there are unusual clinicopathologic features (nonmucosal presentation, HIV-negativity of the patient, and/or EBV-negativity of the neoplastic cells).

\section{Acknowledgement}

We express our appreciation to Deanna Killen for her excellent technical support performing immunohistochemical stains.

\section{References}

1 Delecluse HJ, Anagnostopoulos I, Dallenbach F, et al. Plasmablastic lymphomas of the oral cavity: a new entity associated with the human immunodeficiency virus infection. Blood 1997;89:1413-1420.

2 Gatter K, Warnke R. Diffuse large B-cell lymphoma. In: Jaffe ES, Harris NL, Stein H, Vardiman JW (eds). Pathology and Genetics of Tumours of Haematopoietic and Lymphoid Tissues. IARC Press: Lyon, 2001, pp 171-174.

3 Gaidano G, Cerri M, Capello D, et al. Molecular histogenesis of plasmablastic lymphoma of the oral cavity. Br J Haematol 2002;119:622-628.

4 Chetty R, Hlatswayo N, Muc R, et al. Plasmablastic lymphoma in HIV+ patients: an expanding spectrum. Histopathology 2003;42:605-609.

5 Onciu M, Behm FG, Downing JR, et al. ALK-positive plasmablastic B-cell lymphoma with expression of the NPM-ALK fusion transcript: report of 2 cases. Blood 2003;102:2642-2644.

6 Audouin J, Comperat E, Le Tourneau A, et al. Myeloid sarcoma: clinical and morphologic criteria useful for diagnosis. Int J Surg Pathol 2003;11:271-282.
7 Greipp PR, Raymond NM, Kyle RA, et al. Multiple myeloma: significance of plasmablastic subtype in morphological classification. Blood 1985;65:305-310.

8 Carbone A, Gaidano G, Gloghini A, et al. AIDS-related plasmablastic lymphomas of the oral cavity and jaws: a diagnostic dilemma. Ann Otol Rhinol Laryngol 1999;108:95-99.

9 Flaitz CM, Nichols CM, Walling DM, et al. Plasmablastic lymphoma: an HIV-associated entity with primary oral manifestations. Oral Oncol 2002;38: 96-102.

10 Stewart JM, Krishnamurthy S. Fine-needle aspiration cytology of a case of HIV-associated anaplastic myeloma. Diagn Cytopathol 2002;27:218-222.

11 Aoki $\mathrm{T}$, Okita $\mathrm{H}$, Kayano $\mathrm{H}$, et al. Anaplastic plasmacytoma with malignant pleural effusion lacking evidence of monoclonal gammopathy. Virchows Arch 2002;441:154-158.

12 Hummel M, Anagnostopoulos I, Korbjuhn $\mathrm{P}$, et al. Epstein-Barr virus in B-cell non-Hodgkin's lymphomas: unexpected infection patterns and different infection incidence in low- and high-grade types. J Pathol 1995;175:263-271.

13 Fiorino AS, Atac B. Paraproteinemia, plasmacytoma, myeloma and HIV infection. Leukemia 1997;11: 2150-2156.

14 Kumar S, Kumar D, Schnadig VJ, et al. Plasma cell myeloma in patients who are HIV-positive. Am J Clin Pathol 1994;102:633-639.

15 Colomo L, Loong F, Rives S, et al. Diffuse large B-cell lymphomas with plasmablastic differentiation represent a heterogeneous group of disease entities. Am J Surg Pathol 2004;28:736-747.

16 Grogan TM, Van Camp B, Kyle RA, et al. Plasma cell neoplasms. In: Jaffe ES, Harris NL, Stein H, Vardiman JW (eds). Pathology and Genetics of Tumours of Haematopoietic and Lymphopid Tissues. IARC Press: Lyon, 2001, pp 142-156.

17 Chang CC, McClintock S, Cleveland RP, et al. Immunohistochemical expression patterns of germinal center and activation B-cell markers correlate with prognosis in diffuse large B-cell lymphoma. Am J Surg Pathol 2004;28:464-470.

18 Chan PK, Ng HK, Cheung JL, et al. Survey for the presence and distribution of human herpesvirus 8 in healthy brain. J Clin Microbiol 2000;38:2772-2773.

19 Bergsagel DJ, Finegold MJ, Butel JS, et al. DNA sequences similar to those of simian virus 40 in ependymomas and choroid plexus tumors of childhood. N Engl J Med 1992;326:988-993.

20 Carbone A, Gloghini A, Larocca LM, et al. Expression profile of MUM1/IRF4, BCL-6, and CD138/syndecan-1 defines novel histogenetic subsets of human immunodeficiency virus-related lymphomas. Blood 2001;97: 744-751.

21 Wijdenes J, Vooijs WC, Clement C, et al. A plasmocyte selective monoclonal antibody (B-B4) recognizes syndecan-1. Br J Haematol 1996;94:318-323.

22 Torlakovic E, Torlakovic G, Nguyen PL, et al. The value of anti-pax-5 immunostaining in routinely fixed and paraffin-embedded sections: a novel pan pre-B and B-cell marker. Am J Surg Pathol 2002;26:1343-1350.

23 Sahara N, Takeshita A, Shigeno K, et al. Clinicopathological and prognostic characteristics of CD56-negative multiple myeloma. Br J Haematol 2002;117:882-885.

24 Ruiz-Arguelles GJ, San Miguel JF. Cell surface markers in multiple myeloma. Mayo Clin Proc 1994;69:684-690. 
25 Lima M, Teixeira Mdos A, Fonseca S, et al. Immunophenotypic aberrations, DNA content, and cell cycle analysis of plasma cells in patients with myeloma and monoclonal gammopathies. Blood Cells Mol Dis 2000;26:634-645.

26 de Leval L, Harris NL. Variability in immunophenotype in diffuse large B-cell lymphoma and its clinical relevance. Histopathology 2003;43:509-528.

27 Skinnider BF, Horsman DE, Dupuis B, et al. Bcl-6 and Bcl-2 protein expression in diffuse large B-cell lymphoma and follicular lymphoma: correlation with 3q27 and 18q21 chromosomal abnormalities. Hum Pathol 1999;30:803-808.

28 Colomo L, Lopez-Guillermo A, Perales $\mathrm{M}$, et al. Clinical impact of the differentiation profile assessed by immunophenotyping in patients with diffuse large B-cell lymphoma. Blood 2003;101:78-84.

29 Dogan A, Bagdi E, Munson P, et al. CD10 and BCL-6 expression in paraffin sections of normal lymphoid tissue and B-cell lymphomas. Am J Surg Pathol 2000; 24:846-852.

30 Nutt SL, Thevenin C, Busslinger M. Essential functions of Pax-5 (BSAP) in pro-B cell development. Immunobiology 1997;198:227-235.

31 Barberis A, Widenhorn K, Vitelli L, et al. A novel B-cell lineage-specific transcription factor present at early but not late stages of differentiation. Genes Dev 1990;4:849-859.

32 Ely SA, Knowles DM. Expression of CD56/neural cell adhesion molecule correlates with the presence of lytic bone lesions in multiple myeloma and distinguishes myeloma from monoclonal gammopathy of undetermined significance and lymphomas with plasmacytoid differentiation. Am J Pathol 2002;160:1293-1299.
33 Hammer RD, Vnencak-Jones CL, Manning SS, et al. Microvillous lymphomas are B-cell neoplasms that frequently express CD56. Mod Pathol 1998;11: 239-246.

34 Sekita T, Tamaru JI, Isobe K, et al. Diffuse large B cell lymphoma expressing the natural killer cell marker CD56. Pathol Int 1999;49:752-758.

35 Inaba T, Shimazaki C, Sumikuma T, et al. T-cell associated antigen-positive B-cell lymphoma. Leuk Lymphoma 2001;42:1161-1171.

36 Drach J, Gattringer C, Glassl H, et al. The biological and clinical significance of the KI-67 growth fraction in multiple myeloma. Hematol Oncol 1992;10:125-134.

37 Kawano MM, Mahmoud MS, Ishikawa H. Cyclin D1 and p16INK4A are preferentially expressed in immature and mature myeloma cells, respectively. $\mathrm{Br} \mathrm{J}$ Haematol 1997;99:131-138.

38 Elnenaei MO, Gruszka-Westwood AM, A'Hernt R, et al. Gene abnormalities in multiple myeloma; the relevance of TP53, MDM2, and CDKN2A. Haematologica 2003;88:529-537.

39 Brown RS, Power DA, Spittle HF, et al. Absence of immunohistochemical evidence for human herpesvirus 8 (HHV8) in oral cavity plasmablastic lymphoma in an HIV-positive man. Clin Oncol (R Coll Radiol) 2000;12:194.

40 Webster-Cyriaque J, Edwards RH, Quinlivan EB, et al. Epstein-Barr virus and human herpesvirus 8 prevalence in human immunodeficiency virus-associated oral mucosal lesions. J Infect Dis 1997;175:1324-1332.

41 Cioc AM, Allen C, Kalmar JR, et al. Oral plasmablastic lymphomas in AIDS patients are associated with human herpesvirus 8. Am J Surg Pathol 2004;28: 41-46. 